\title{
Las características del emprendedor mexicano: estudio de caso de Lean Startups México
}

\section{Characteristics of the Mexican entrepreneur: case study Lean Startups Mexico}
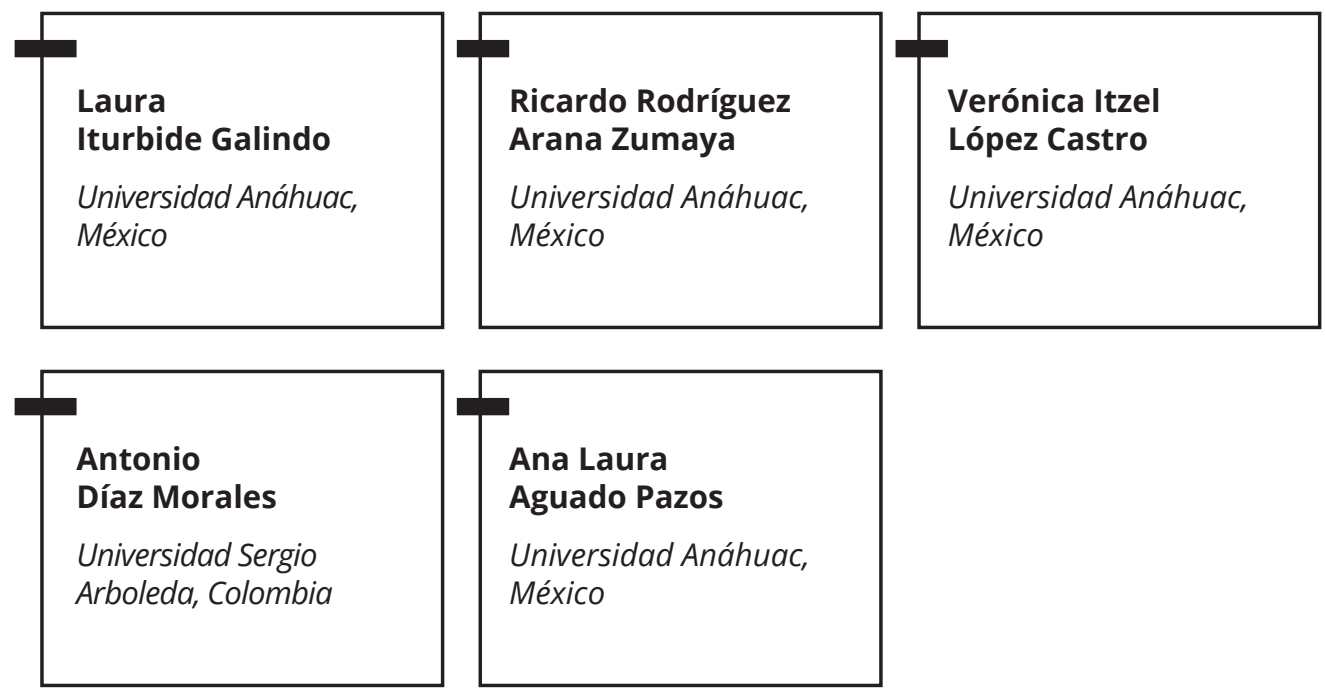

Recibido: 11 de agosto de 2020.

Aprobado: 29 de octubre de 2020. 


\section{Resumen}

El perfil del emprendedor ha sido un tema ampliamente discutido en la literatura, sin embargo, es escasa aquella que trata de las características del emprendedor latinoamericano debido a que se centra principalmente en otras regiones. Este estudio compara qué talentos están presentes en aquellos que logran establecer una empresa frente a los que no lo logran. Para ello, se tomó una muestra de 33 líderes de equipos emprendedores, de una población total de 60, pertenecientes a cuatro entidades de México. Todos ellos participaron en el programa Lean Startups México y fueron intervenidos con la metodología Lean Startup. Se levantó un inventario creado para la metodología Talent Motivation Analysis (TMA) y, finalmente, se hizo un análisis descriptivo de los resultados, concluyendo que hay talentos sobresalientes propios del emprendedor, sin importar su zona geográfica, y otros en los que el entorno marca la diferencia. Adicionalmente se concluyó que, a pesar de haber pasado por el proceso de formación y mentoría de la metodología Lean Startup, la mayoría de los talentos de los emprendedores, especialmente la orientación al cliente y la innovación, mostraron un impacto mínimo e incluso una reducción, en contraposición a los resultados que el método propone.

Palabras clave: emprendimiento; innovación; perfil.

Clasificación JEL: M10, M13.

\section{Abstract}

The entrepreneurial profile has been a widely discussed topic in the literature, however, there is not enough literature with the characteristics of the Latin American entrepreneur, due to its main focus on other regions. This study compares what talents are present in entrepreneurs who manage to establish a company, versus those who fail. To do this, a sample of 33 leaders of entrepreneurial teams was selected, from four entities in Mexico, that belong to a total population of 60, who participated in the Lean Startups Mexico program and were trained with Lean Startup methodology. They filled an inventory created for the Talent Motivation Analysis (TMA) methodology, and finally, a descriptive analysis of the results was carried out, concluding that there are outstanding talents typical of entrepreneurs, regardless of their geographical area, others where the environment makes a difference and some more that are not necessarily present in their innate abilities. In addition to this, it was concluded that, despite having gone through the training and mentoring process in the Lean Startup methodology, most of these entrepreneurial talents, especially customer orientation and innovation, showed minimal or even a reduction in the impact, in opposition to what proposed method results.

Keywords: entrepreneurship; innovation; profile.

JEL Classification: M10, M13. 


\section{Introducción y antecedentes}

El emprendimiento ha sido considerado como una «megatendencia» 1 que, especialmente en la última década, ha tomado un nuevo cariz. Así, de un emprendedor más arriesgado y mítico que se generó durante el siglo XIX y parte del XX, hoy toma su lugar uno más humanizado (Rodríguez, 2014).

La connotación actual del término emprendimiento fue dada a conocer por Schumpeter en 1911, quien presentó a los emprendedores como motores de cambio para el desarrollo y la implementación de ideas (Lindner, 2018). Desde ese entonces se destacaba también el papel fundamental de las nuevas empresas para la generación de nuevos productos y servicios, como resultado de la introducción de innovaciones (Schumpeter, 1934, 2013). En este orden de ideas, se puede definir al emprendedor como aquella persona que crea organizaciones (Gartner, 1988).

Adicionalmente, la reducción del papel del Estado como generador de empleos ha destacado el rol del emprendedor y de las nuevas empresas porque su impacto en la economía es dinámico, además de estratégico, a tal punto que el emprendimiento, hoy en día, es muy valorado dentro del currículo educativo por su potencial para el desarrollo económico (Uriguen, Pizarro y Cedeño, 2018). Dadas las bondades antes mencionadas, el emprendimiento destaca como uno de los campos en la educación con mayor crecimiento (Solomon y Fernald, 1993).

Sin embargo, hasta hace apenas unos años, el emprendimiento no solo sonaba como algo distante en México, sino en el mundo entero, y se reservaba únicamente a los pocos capaces de atreverse. Por ello, actualmente se ha gestado una verdadera revolución en la visión del tema, un giro de 180 grados en torno a este, cuyo significado es ya mucho más que comenzar un nuevo negocio, sino que es la oportunidad de convertir a las personas en seres más creativos, proactivos e innovadores (Iturbide, 2018).

A pesar de esta nueva visión, el emprendedor y su circunstancia apenas comenzaron a ser tratados en estudios con rigor metodológico hace algunos años. Como

\footnotetext{
${ }_{1}^{1}$ Megatendencia es una dirección que toman simultáneamente varios indicadores de las sociedades (tecnología, sistemas de producción, nuevos productos, preferencias de consumo, comportamientos, etcétera), cuyo impacto es perceptible para un segmento importante durante un largo tiempo. De hecho, muchas megatendencias se convierten en parte de la cotidianidad (Grupo de Desarrollo Regional del Tecnológico de Monterrey, 2018).
} 
resultado, se empezó a desatar una ola de estudios globales relacionados, entre los cuales el más destacado es el Global Entrepreneurship Monitor (GEM, 2019), cuyo objetivo es medir la intención emprendedora mediante la comparación de emprendedores de diferentes países.

También se ha comenzado a generar una vasta literatura científica en temas tales como oportunidad emprendedora, generación de valor o perfil emprendedor; sin embargo, la mayoría de los textos más citados no están centrados en países de habla hispana y menos aún de América Latina, sino en Estados Unidos y Europa (Luor, Lu, Yu y Chang, 2014).

Dicho lo anterior, una aportación fundamental es la generación de estudios específicos para los emprendimientos de la región latinoamericana, en particular aprovechando la reciente ola de programas emprendedores que se ha financiado en los últimos años.

El principal objetivo del presente estudio es estudiar el perfil del emprendedor mexicano comparando el impacto de sus atributos o talentos cuando se somete a un grupo de emprendedores de un programa específico y a una intervención experimental con un proceso de asesoría y formación.

Para ello, se ejecutó un estudio cuantitativo de corte descriptivo, cuyo objetivo es comparar dos grupos de estudio seleccionados por conveniencia, a través de una muestra obtenida a partir de la población total del programa Lean Startups México (2018). Ambos se sometieron a una intervención en la primera etapa, que incluyó formación y asesoría para descubrir al cliente al que se atendería con su emprendimiento; solo el segundo grupo fue sometido a una segunda etapa en la cual se le brindó un proceso de asesoría para lograr la construcción de la compañía. Para realizar el comparativo se levantó un inventario a ambos grupos y se compararon los resultados.

El artículo comienza con una revisión de la literatura, seguido de la descripción de la metodología empleada, los resultados obtenidos y las conclusiones, así como futuras líneas de investigación.

\section{Revisión de la literatura}

Un emprendedor no solo tiene que hacer frente a sus propias limitantes, como la falta de tiempo, habilidades o recursos, sino también a aquellas inherentes al entorno en el que se mueve, como por ejemplo, trámites y burocracia (Vázquez-Parra, 2018). 
Según Alan Carsrud, profesor de emprendimiento de la Äbo Akademi, universidad sueca ubicada en Finlandia, se piensa que el uso de la tecnología es la clave para que un negocio tenga alto crecimiento y sea sostenible, cuando en realidad lo más importante es entender las habilidades y motivadores que debe tener un emprendedor para llevar a cabo su proyecto (López, 2019).

Los emprendedores comienzan con la ilusión de ser exitosos pero, desafortunadamente, no todos logran que su startup lo sea; sin embargo, aquellos que no alcanzan el éxito normalmente no se dan por vencidos, sino que intentan comenzar un nuevo emprendimiento aplicando una estrategia diferente a la utilizada la primera vez (Dorf, 2015).

En este sentido, se puede decir que, para iniciar, son varios los motivadores que guían a un emprendedor, como la necesidad de tener logros independientes o bien los objetivos económicos; además, por naturaleza es un ser transformador, capaz de percibir oportunidades en su entorno; su capacidad creativa es una característica diferenciadora, pero existen muchas más; otros atributos están asociados con su tenacidad, equilibrio emocional y autocrítica, que lo llevan a diferenciar y calibrar riesgos, aceptar errores y aprender de ellos (Montoya, Correa y Mejía, 2009).

Entonces, entender el emprendimiento bajo diferentes perspectivas se vuelve imperativo, ya que inducir el potencial emprendedor involucra no solo conceptos, procesos o estructuras para la creación de una empresa, sino también una serie de actitudes que formen esa mentalidad emprendedora (Kurakto, Fisher y Audretsch, 2020).

Por eso, al cuestionamiento de si el emprendedor se hace o nace podría responderse que es una mezcla de ambas (Montoya, Correa y Mejía, 2009). A pesar de los talentos innatos, sí es posible motivar la formación de tales actitudes en los estudiantes, es decir, desarrollar las competencias requeridas o deseadas en los emprendedores (Uriguen, Pizarro y Cedeño, 2018).

\section{Enfoques para analizar el perfil del emprendedor}

Joseph Schumpeter $(1934,2013)$, pionero de los conceptos de emprendimiento, determinaba que la verdadera función del emprendedor era la toma de iniciativas. Si esto se circunscribe a la esencia psicológica del emprendedor, las competencias relacionadas con esta son la capacidad de análisis y la creatividad. 
Sin embargo, diversas escuelas de pensamiento del emprendimiento han llegado a determinar otros elementos importantes en el perfil emprendedor, desde perspectivas psicológicas hasta etológicas.

\section{a) El enfoque etológico}

De acuerdo con este, un emprendedor está influido por su entorno (hábitat), pero también por características internas intrínsecas y otras adquiridas (competencias), así como por una serie de estímulos y motivadores que mejoran su condición de vida y entorno.

Este enfoque parte de interrogantes que se examinan de acuerdo con diversas perspectivas. En la literatura se responden estudiando los estímulos tanto internos como externos que desatan ciertos comportamientos en el emprendedor, su interacción con el entorno, su proceso de adaptación y sus resultados (Timbergen, 1963). En este sentido, se le puede entender como un sujeto que busca adquirir nuevo conocimiento con la intención de lograr la adaptación al entorno que, desde esta perspectiva, tiene un papel fundamental en la generación de habilidades cognoscitivas (Montoya, Correa y Mejía, 2009).

\section{b) El enfoque psicológico}

Esta perspectiva incluye la postura de la escuela del comportamiento humano y psicológico, que se enfoca en comprender el proceso de creación de empresas mediante el análisis de la iteración, es decir, el continuo de fracasos y éxitos. Así, al emprendedor le gusta asumir riesgos y controlar su destino (Gartner, 1988). Por esta razón, una de las características básicas de los emprendedores, de acuerdo con esta corriente, es la tolerancia al fracaso (Fuentelsaz y Montero, 2015).

Por otra parte, desde la perspectiva psicocognitiva, la educación, habilidades e interés por el pensamiento abstracto, así como por la curiosidad de encontrar soluciones generales a problemas (Koellinger, 2008) pueden tener como resultado el descubrimiento de oportunidades y la oferta de productos o servicios innovadores. Esto no es sencillo y se ve condicionado por la información previa de los individuos (Venkataraman, 1997).

Montoya, Correa y Mejía (2009) resumen en cinco grupos las características que se han identificado como básicas en la literatura para definir el perfil psicológico de 
los emprendedores. Estos son: a) deseo de independencia; b) gusto por el riesgo; c) elevada necesidad de logro; d) alta necesidad de competencia y e) preferencia por la innovación.

En resumen, podría decirse que el emprendedor es una persona que puede describirse no solo por una serie de atributos psicológicos, sino por sus procesos cognitivos, que a su vez se activan ante ciertas circunstancias (Shaver y Scott, 1991).

\section{La metodología TMA empleada para el análisis de talentos del perfil emprendedor}

Con el ánimo de poder efectuar una medición de los talentos o atributos del perfil emprendedor, a partir de un inventario de los mismos, se han realizado diversos análisis y se aplicaron varios cuestionarios, en su mayoría desde una perspectiva de enfoque de competencias (Lackéus, 2015; Lindner, 2018). Sin embargo, existen algunos inventarios que no han sido utilizados para estos fines, tal es el caso de la metodología Talent Motivation Analysis (TMA, por sus siglas en inglés).

La TMA nació en Holanda y actualmente tiene una gran presencia en Europa y Asia. Se basa en la teoría del psicólogo Henry Murray (1938), quien sostenía que todos los seres humanos responden a los mismos motivadores, la diferencia consiste en que algunos los tienen en mayor medida que otros.

La esencia de la metodología es sencilla y poderosa, ya que trabaja con la naturaleza del individuo de forma personalizada, tratando de entenderla y así poder determinar categorías de talentos, los cuales se miden a través de preguntas contenidas en un estudio psicométrico de 170 reactivos con el análisis ipsativo. Este permite asegurar la consistencia de los resultados. Además, se diferencia de otros exámenes psicométricos porque realiza preguntas enfocadas en el motivador del individuo, es decir, a nivel personal y no únicamente profesional (Martínez-Marbán, 2018).

En este sentido, la metodología sostiene que, para poder seleccionar, desarrollar y evaluar a una persona en función de su comportamiento, es importante considerar los factores que lo afectaron (Martínez-Marbán, 2018). Para el desarrollo de competencias, la herramienta combina el enfoque etológico con el psicológico (ver figura 1). 
Figura 1. Metodología TMA: del impacto al rendimiento
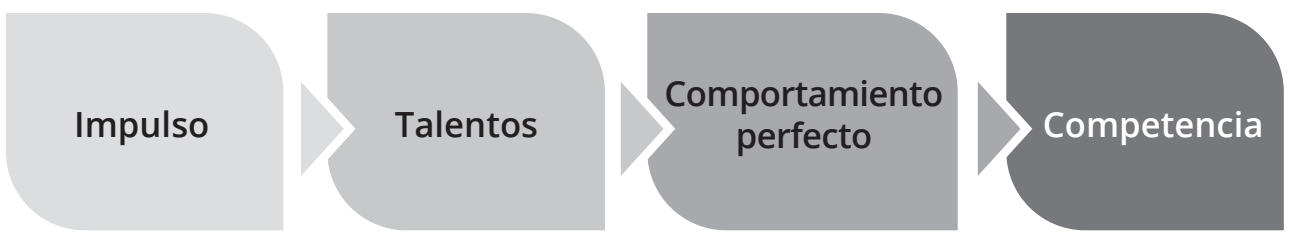

Fuente: elaboración propia, con base en Martínez-Marbán (2018).

Para entender los pasos subsecuentes descritos en la figura 1, se desarrolló un ejemplo (ver figura 2). Como se puede observar, primero es necesario identificar el impulso; es decir, encontrar el patrón inconsciente de necesidades con ideas relacionadas, temores y deseos. Entendiendo los motivadores, se determinan los talentos que distinguen a una persona de las demás. Estos llevan a que el individuo tenga un comportamiento preferido, es decir, que se sienta más cómodo actuando de una u otra forma y pueda desarrollar una determinada competencia. A continuación, se muestra un ejemplo para clarificar la comprensión de lo mencionado.

Figura 2. Ejemplo del impacto al rendimiento

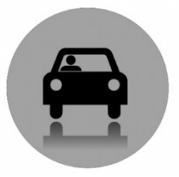

IMPULSO

Alta necesidad de cumplimiento y respeto.

Deseo de ser visto y reconocido.

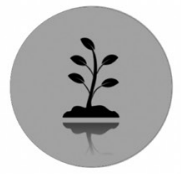

TALENTOS

Presentable y orgulloso.

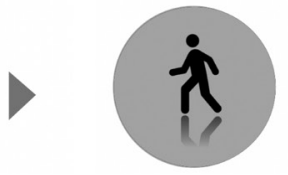

COMPORTAMIENTO PREFERIDO

Prefiere lucir bien y presentable y le atribuye mucha importancia a su estado personal y a ganar reconocimiento.

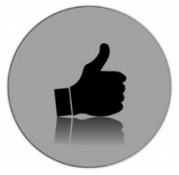

COMPETENCIA

Conducta: dar una buena primera impresión y lograr mantenerla a lo largo del tiempo.

Fuente: elaboración propia, con base en Martínez-Marbán (2018).

El comportamiento de una persona, entonces, es lo que los demás observan y, con esta metodología (ver figura 3), pueden distinguirse tres factores influyentes que lo afectan: motivadores y talentos, competencias y capacidades y entorno (Van IJzendoorn, Van Weer, Müller y Blom, 1996). Es decir, la metodología base con la cual se desarrolló el inventario que se aplica en la metodología TMA cumple con dos enfoques, tanto el psicológico como el etológico. 
Figura 3. Proceso de la metodología TMA

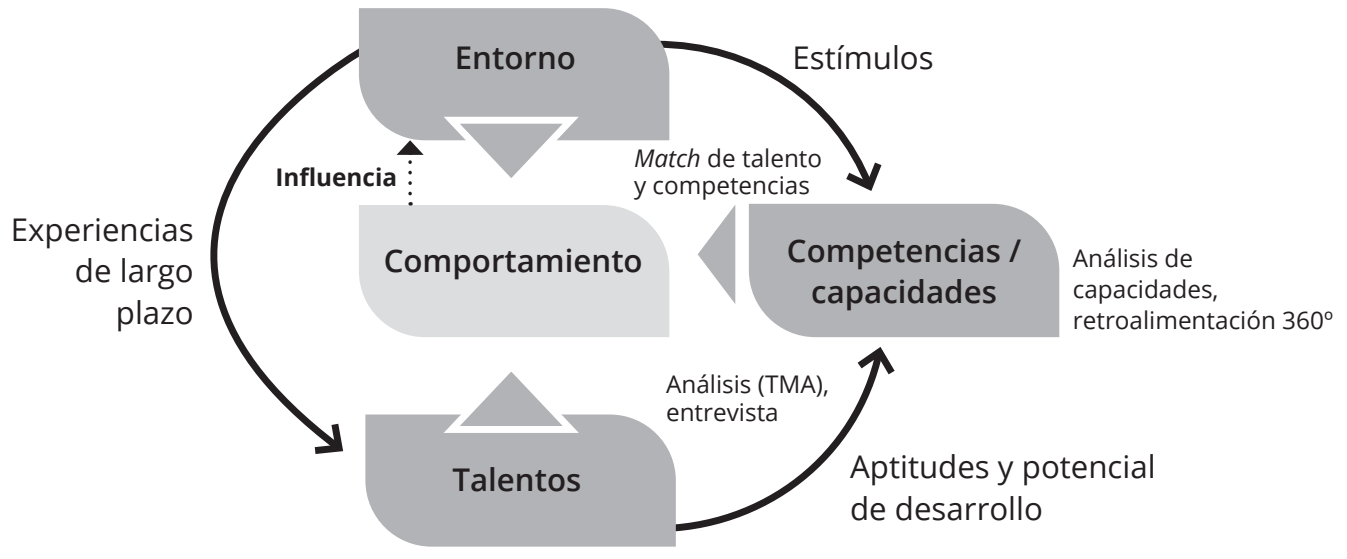

Fuente: elaboración propia, con base en Martínez-Marbán (2018).

En este sentido, se puede destacar que las competencias son operadas por el comportamiento, es decir, la aptitud de una persona para su desarrollo. Por lo tanto, que una competencia pueda desarrollarse fácilmente significa que, de acuerdo con los talentos del individuo, le costará menos trabajo y, por lo tanto, le consumirá menos energía desarrollarla. Cuanto más se acerca el comportamiento a una competencia, mayor será la correlación entre el potencial de desarrollo y la manifestación de tal comportamiento (Van IJzendoorn et al., 1996).

\section{Diferencias regionales en el perfil emprendedor}

Otra perspectiva del perfil emprendedor es el análisis de diferencias regionales en el país. Este no ha sido previamente explorado en la literatura debido a que la mayoría se centra en las experiencias de países europeos y Estados Unidos (Luor et al., 2014). En este sentido, es importante comprender las características de las regiones de nuestro país.

De acuerdo con el Censo Económico 2020, las tres entidades que concentran el mayor número de unidades económicas son el Estado de México, la Ciudad de México y Veracruz. De las 6373169 unidades económicas, 46.8 \% se dedica al comercio, $39 \%$ a servicios no financieros y el diferencial a la manufactura $u$ otras actividades económicas. Sin embargo, esta composición se aprecia muy distinta al analizar las vocaciones regionales por entidad, en el norte y zona del Bajío, predominantemente 
de corte industrial, y de servicios y comercio en el centro y sur, salvo contadas excepciones (INEGI, 2020).

En este sentido, podemos destacar que las entidades con vocación de corte industrial y que se encuentran por arriba de la media nacional (45.6 \%) son las siguientes: Campeche, Tabasco, Coahuila, San Luis Potosí, Aguascalientes, Sonora, Tlaxcala, Guanajuato, Zacatecas, Querétaro, Chihuahua, Tamaulipas, Puebla, Durango, Baja California, Morelos, Estado de México, Hidalgo y Nuevo León (en ese orden). En sentido contrario, las entidades ubicadas por debajo de la media en cuanto al corte industrial presentan una vocación mayoritariamente de servicios y comercio; tal es el caso de Veracruz, Jalisco, Oaxaca, Yucatán, Michoacán, Sinaloa, Chiapas, Guerrero, Colima, Baja California Sur, Ciudad de México, Nayarit y Quintana Roo (INEGI, 2020).

Asimismo, los emprendedores de cada entidad se enfrentan a diversos retos locales cuando intentan abrir y operar una empresa, los cuales no son necesariamente iguales en cada entidad, de tal modo que el tiempo de apertura, así como el costo o la cantidad de trámites que se requieren pueden variar de una entidad a otra según el informe anual Doing Business in 2020 (Banco Mundial, 2020).

No es de extrañar que los emprendedores de cada región presenten diferencias en sus especializaciones y hasta en conocimientos y habilidades desarrolladas. La dificultad de su estudio radica en que los reportes consolidados que se generan suelen ser de carácter nacional o local, y no hay un estudio periódico que mida estas diferencias por entidad. El más destacado es el GEM, sin embargo, además de ser un consolidado y solo contar con algunos reportes estatales, no tiene capítulos en todas las localidades, por lo tanto, solo ofrece mediciones por entidad. Adicionalmente cabe destacar que el GEM se enfoca en la intención emprendedora, características sociodemográficas del emprendedor y el contexto de emprendimiento, más que en los rasgos psicológicos del emprendedor (GEM, 2015).

Debido a lo anterior, es escasa la información sobre el contexto local por entidad referente a las características propias del emprendedor.

En los siguientes apartados se describen los objetivos del estudio, se formula la pregunta de investigación, se expone la metodología utilizada y se presentan los principales resultados, conclusiones y futuras líneas de investigación que arrojó este estudio. 


\section{Objetivos y preguntas de investigación}

El objetivo principal de este estudio fue comprender la variación en los talentos (perfil/ características) con los que cuenta un emprendedor del programa Lean Startups México y su efecto, posterior al programa. La pregunta de investigación es la siguiente: ¿qué talentos se modifican en los emprendedores líderes de cada equipo que pasan a la etapa de construcción de la compañía contra los que se quedan en la etapa inicial de descubrimiento del cliente, dentro del programa Lean Startups México?

\section{Metodología}

Para los fines de esta investigación, se recurre al análisis descriptivo con un enfoque cuantitativo; es decir, el enfoque se propone describir las características de la población objetivo. Inicialmente se realizó el cálculo de la muestra requerida tomando como población total a los líderes de los 60 equipos participantes del programa Lean Startups México (edición 2018); posteriormente, se procedió a levantar su inventario de talentos según el modelo desarrollado por TMA y, finalmente, se llevó a cabo el análisis descriptivo de los resultados. A continuación se presentan las características del programa del que se obtuvieron los participantes del estudio, el cálculo de la muestra, el detalle de la sección de los ítems aplicados del cuestionario, el proceso de levantamiento de información y las características de la muestra.

\section{El programa Lean Startups México}

En 2015, la Universidad Anáhuac México, con apoyo del Instituto Nacional del Emprendedor e inversionistas privados, lanzó el programa Lean Startups México, la primera iniciativa nacional que buscaba difundir la metodología Lean Startup en todo el país. Esta primera emisión fue tan exitosa que logró inscribir a 696 equipos de emprendedores para seleccionar un grupo final de solo 30 proyectos. Se formaron equipos que recibieron mentoría de Bob Dorf, coautor de la metodología, y lograron presentar su idea de negocio a un grupo de inversionistas potenciales después de diez intensas semanas de trabajo.

El programa marcó un hito en el ecosistema. Como resultado, en 2018, el Banco Interamericano de Desarrollo a través del IDB Lab, aprobó una segunda emisión a la Aceleradora de Negocios IDEARSE-Anáhuac para ejecutar el programa, pero en 
esta ocasión con mayor alcance no solo en cuanto al número de equipos, sino en lo referente a la distribución geográfica y, sobre todo, en lo que toca al nivel de profundización de la metodología (https://leanstartupsmexico.com.mx).

Esta nueva emisión contó con 60 personas, seleccionadas por un comité evaluador, en cuatro entidades del país (Estado de México, Hidalgo, Mérida y San Luis Potosí), en total 15 equipos participantes por cada entidad.

Se hizo una intervención inicial a los 60 equipos con la metodología Lean Startup, mediante formación y mentoría; los participantes sometieron sus proyectos emprendedores en la primera parte de la metodología, conocida como Descubrimiento del cliente, con el objetivo de validar si sus suposiciones de mercado eran correctas. A continuación, solo los mejores 24 equipos fueron seleccionados por un segundo comité evaluador. En el siguiente paso estos fueron intervenidos mediante asesoría para prepararse para el lanzamiento de su organización. Es decir, se sometieron a la segunda etapa de la metodología denominada Construcción de la compañía.

Este tratamiento por etapas se apega a la recomendación de Blank y Dorf (2012) de no lanzar la compañía al mercado hasta no haber validado en una primera etapa que existe un mercado para la idea y que el emprendedor ha podido invalidar sus propias hipótesis y suposiciones respecto al modelo de negocio.

\section{Determinación del tamaño de la muestra}

Cada uno de los 60 equipos participantes del programa (15 de cada entidad), contaba con tres o cuatro participantes inscritos, respetando la recomendación de Blank y Dorf (2012) según la cual uno de ellos era el líder o visionario que aportaba la idea de proyecto; otro integrante fungía como el técnico del proyecto (hacker), además de uno o dos integrantes especialistas en mercadotecnia y ventas.

Con el objetivo de obtener el número de inventarios representativos de la población total, se procedió a calcular el tamaño de la muestra. La población total considerada incluye a todos aquellos líderes de equipos o visionarios participantes del programa Lean Startups México durante la segunda emisión de 2019, un total de 60 líderes de equipo, uno por cada uno. La razón por la cual solo se consideró a los líderes de equipos y no a todos los participantes del programa es porque ellos son quienes, además de haber registrado el proyecto en el programa como fundadores, detonan la idea emprendedora y se interesan por dar inicio al emprendimiento, armando posteriormente el equipo emprendedor (Blank y Dorf, 2012). 
Por conveniencia, es decir, por ser con la que se contaba a la mano, estas 60 personas se consideraron como la población total. A partir de ella, se tomó una muestra representativa, calculada con un margen de error de $10 \%$ y un nivel de confianza de $90 \%$, que son opciones típicas para obtener una muestra adecuada. Como resultado, la conclusión fue que, para poder llevar a cabo el estudio se necesitaba un total mínimo de 31 líderes de equipo participantes que respondieran el cuestionario.

De acuerdo con la fórmula detallada por Pickers (2015) para el cálculo de muestras, esta, integrada por 31 líderes, se calculó como se indica a continuación:

$$
\begin{aligned}
& n=\frac{Z^{2}(p * q)}{e^{2}+Z^{2}\left(p^{*} q\right) / N} \\
& n=\frac{(1.645)^{2}(0.5 * 0.5)}{(0.1)^{2}+(1.645)^{2}(0.5 * 0.5) / 60} \\
& n=\frac{(2.706)(0.25)}{(0.1)+(2.706)(0.25) / 60} \\
& n=\frac{0.6765}{0.1+0.0113} \\
& n=\frac{0.6765}{0.0212} \\
& n=31
\end{aligned}
$$

Donde:

$\mathrm{n}=$ tamaño de la muestra.

$\mathrm{N}=$ tamaño de la población.

$\mathrm{Z}$ = valor correspondiente $a$ la distribución de Gauss 1.645 para a =.10, llamado también nivel de confianza.

$p$ = proporción aproximada del fenómeno de estudio en la población de referencia.

$q$ = la proporción de la población de referencia que no presenta el fenómeno en estudio ( $1 \mathrm{p})$.

$\mathrm{e}=$ término de error. 
Cabe señalar que, a pesar de que el número idóneo de cuestionarios a levantarse era de 31, se logró obtener un total de 33, lo cual permitió lograr un mayor número de resultados del calculado inicialmente.

\section{El inventario TMA y la selección de los ítems aplicados}

El inventario levantado de la muestra calculada fue el que se desarrolló por la metodología TMA, es decir, no se diseñó un cuestionario para este estudio, sino que se utilizó uno ya existente y probado para estos fines y se consideró que era idóneo. La razón es que este tomó como base tanto el enfoque etológico como el psicológico, es decir, además de las características psicológicas de los participantes se enfocó en su entorno. Asimismo, la diferencia principal respecto a otros cuestionarios es que realiza preguntas enfocadas al motivador del individuo no solo en el nivel profesional sino también en el personal (Martínez-Marbán, 2018).

A fin de seleccionar los talentos, que fueron plasmados en el cuestionario a los emprendedores, se siguieron dos pasos. El primero, una entrevista a profundidad con la especialista, Ing. Martínez Marbán, responsable de TMA en México, con la finalidad de conocer en detalle la metodología.

El segundo consistió en que esta información fue un insumo para la selección de los reactivos a ser considerados, tomando como base la literatura y con la participación de un grupo seleccionado de expertos de emprendimiento y ejecutores del programa para, a su vez, poder determinar los talentos predominantes del emprendedor que deberían evaluarse. Resultaron ser los siguientes, tomados expresamente del Documento Oficial de Certificación TMA (Martínez-Marbán, 2018): decisión, discernimiento, adaptabilidad, capacidad de aprendizaje, conciencia social, iniciativa, coraje, espíritu innovador, control del progreso, orientación al cliente.

\section{Levantamiento del inventario}

Se invitó a los líderes de los 60 equipos participantes a responder en línea un cuestionario de 170 preguntas, enfocadas principalmente en sus preferencias sobre los talentos analizados. Se llevó asimismo seguimiento telefónico con la finalidad de lograr obtener al menos los 31 cuestionarios definidos como mínimo en el cálculo 
de la muestra. Se obtuvieron 33 cuestionarios, 11 de los líderes de equipo que solo cursaron la primera etapa del programa (Descubrimiento del cliente) y 22 de los líderes de equipo que cursaron ambas etapas (Construcción de la compañía). Lo anterior permitió que posteriormente se compararan los resultados de ambos grupos. Se denominó Grupo 1 a los participantes (22 personas) intervenidos con formación y mentoría en una segunda etapa (Construcción de la compañía); el Grupo 2 incluyó a los participantes (11 personas) intervenidos solo en la primera etapa del proceso.

Las preguntas que se formularon en el cuestionario fueron muy sencillas, con la finalidad de enfrentarlos a dos tipos de cuestionamientos:

1. Elegir la opción con la que se sintieran más identificados (1 de 2).

2. Ordenar las opciones con las que se identificaba el individuo en forma decreciente, de más a menos.

Este tipo de cuestionamiento corresponde al análisis ipsativo, razón por la cual el cuestionario fue repetitivo, si bien cada pregunta era diferente, a fin de lograr consistencia en los resultados. Una vez que se llevó a cabo el levantamiento, se envió el cuestionario a cada uno de los líderes de equipos para validar el resultado obtenido en cada uno de ellos. El reconocimiento fue, en el peor de los casos, del $95 \%$ de los ítems preguntados, es decir, la variación de respuestas fue mínima y solo con fines de confirmación.

\section{Características de la muestra}

Los líderes de equipo que respondieron el cuestionario tenían 33 años en promedio y habitaban en cuatro entidades del país: Estado de México, Hidalgo, San Luis Potosí y Yucatán. A continuación, se presenta una gráfica (ver gráfica 1) con el detalle de sus características sociodemográficas. 
Gráfica 1. Características sociodemográficas de los participantes

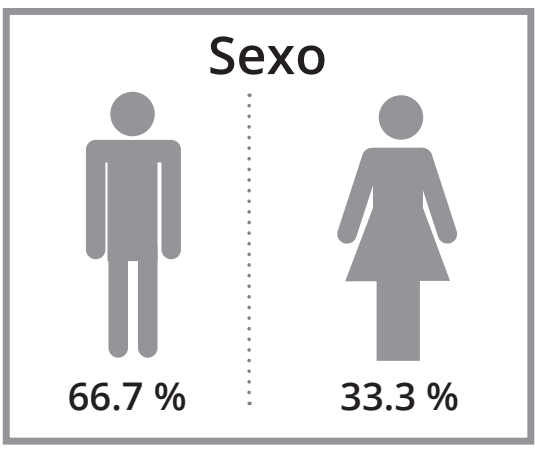

\section{Edad promedio} 33 años

\section{Localidad de origen}

Hidalgo

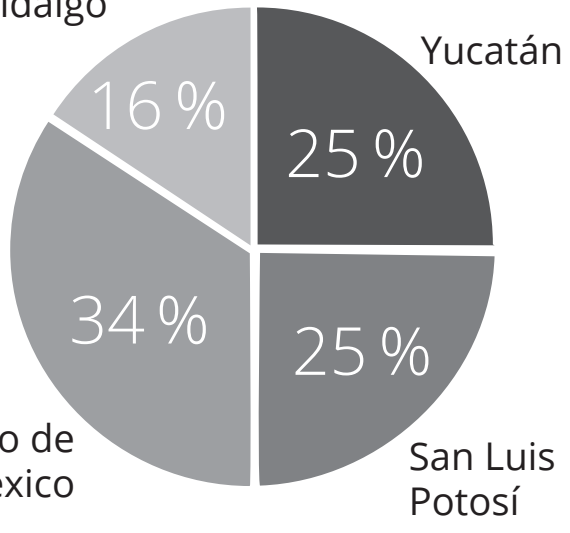

Fuente: elaboración propia con base en información del programa Lean Startups México (2018).

Adicionalmente, se detallan los giros de los emprendimientos de los 33 líderes de equipos que participaron en el estudio, en su mayoría proyectos de base tecnológica, enfocados primordialmente en Edutech y soluciones digitales para negocios; cabe señalar que los proyectos reclutados por cada entidad tenían como condición ser digitales y pertenecer a giros que apoyasen el desarrollo y las vocaciones locales (ver tabla 1).

Tabla 1. Giro de los proyectos participantes

\begin{tabular}{|l|c|c|c|c|c|}
\hline Giro & $\begin{array}{c}\text { Estado de } \\
\text { México }\end{array}$ & Hidalgo & $\begin{array}{c}\text { San Luis } \\
\text { Potosí }\end{array}$ & Yucatán & $\begin{array}{c}\text { Cantidad de equi- } \\
\text { pos participantes }\end{array}$ \\
\hline Agrotech & 0 & 0 & 3 & 0 & 3 \\
\hline Edutech & 4 & 2 & 2 & 1 & 9 \\
\hline Edutech/Healthtech & 0 & 0 & 0 & 2 & 2 \\
\hline Healthtech & 1 & 0 & 1 & 1 & 3 \\
\hline
\end{tabular}


(continuación)

\begin{tabular}{|l|c|c|c|c|c|}
\hline Giro & $\begin{array}{c}\text { Estado de } \\
\text { México }\end{array}$ & Hidalgo & $\begin{array}{c}\text { San Luis } \\
\text { Potosí }\end{array}$ & Yucatán & $\begin{array}{c}\text { Cantidad de equi- } \\
\text { pos participantes }\end{array}$ \\
\hline Matchmaking & 2 & 1 & 0 & 0 & 3 \\
\hline $\begin{array}{l}\text { Soluciones digitales } \\
\text { para negocios }\end{array}$ & 0 & 4 & 3 & 0 & 7 \\
\hline Sustentabilidad & 5 & 0 & 0 & 0 & 5 \\
\hline Transporte & 0 & 0 & 0 & 1 & 1 \\
\hline Total & $\mathbf{1 2}$ & $\mathbf{7}$ & $\mathbf{9}$ & $\mathbf{5}$ & $\mathbf{3 3}$ \\
\hline
\end{tabular}

Fuente: elaboración propia con base en información del programa Lean Startups México (2018).

\section{Resultados}

Los resultados que se muestran a continuación corresponden a todas las personas encuestadas (33 en total); tanto aquellas que pasaron a la etapa de Construcción de la compañía (22 de los 33 inventarios), así como quienes no pasaron a dicha etapa (11 de los 33 inventarios); es decir, que se quedaron en la etapa inicial de Descubrimiento del cliente.

Es importante destacar que la diferencia entre ambos grupos radicó en que los integrantes del Grupo 1 (22 participantes) sí fueron intervenidos en una segunda etapa (Construcción de la compañía) mientras que los del Grupo 2 (11 participantes) solo participaron en la primera etapa del programa (Descubrimiento del cliente). Por lo tanto, a continuación se presentan los resultados desagregados, comparando un grupo con otro, es decir, el Grupo 1 intervenido en una segunda etapa contra el Grupo 2 que no fue intervenido en la misma.

Se reflejan así los resultados obtenidos del grupo completo y los segmentados. Para los fines de este estudio se define como Grupo 1 al formado por los 22 líderes de equipo que pasaron a la siguiente etapa de Construcción de compañía y como Grupo 2 a los 11 líderes de equipo que se quedaron en la etapa de Descubrimiento del cliente, para así poder observar sus diferencias. 


\section{Resultados obtenidos}

Se puede observar que al comparar el Grupo 1 versus el Grupo 2 (ver tabla 2), los tres talentos predominantes son similares, en principio, para ambos grupos; sin embargo, el resto de los atributos presentan mayores diferencias.

Los tres atributos con puntajes más altos fueron (Grupo 1 con 95 \% versus Grupo 2 con $100 \%$ ) y discernimiento (Grupo 1 con $95 \%$ versus Grupo 2 con $100 \%$ ) y adaptabilidad (Grupo 1 con 77 \% versus Grupo 2 con $73 \%$ ).

No obstante, los porcentajes más bajos resultaron comunes para ambos grupos, y se refirieron a control de progreso y orientación al cliente, con porcentajes incluso más reducidos en ambos talentos para el Grupo 2, que se quedó en la etapa inicial de Descubrimiento del cliente (Grupo 1 con 28 \% y Grupo 2 con $27 \%$ ).

Sin embargo, como lo muestra la tabla 2, comparando el Grupo $1(n=22)$ y el Grupo 2 $(n=11)$, el primero muestra mayores porcentajes en cuatro talentos (conciencia social, control del progreso, adaptabilidad y orientación al cliente), mientras que en el segundo lo hace en seis (iniciativa, decisión, discernimiento, coraje, capacidad de aprendizaje y espíritu innovador). Cabe resaltar que el talento denominado «espíritu innovador» es el que muestra una variación mayor, con un total de $23 \%$ de reducción del Grupo1 contra el Grupo 2, que solo participó en la primera etapa del programa.

Tabla 2. Análisis comparativo de talentos: Grupo 1 versus Grupo 2

\begin{tabular}{|l|c|c|c|}
\hline & 22 (en \%) & 11 (en \%) & $\begin{array}{l}\text { Diferencia en puntos } \\
\text { porcentuales }\end{array}$ \\
\hline Decisión & 95 & 100 & -5 \\
\hline Discernimiento & 95 & 100 & -5 \\
\hline Adaptabilidad & 77 & 73 & 4 \\
\hline Capacidad de aprendizaje & 73 & 82 & -9 \\
\hline Conciencia social & 72 & 63 & 9 \\
\hline Iniciativa & 68 & 72 & -4 \\
\hline Coraje & 64 & 73 & -9 \\
\hline Espíritu innovador & 41 & 64 & -23 \\
\hline Control del progreso & 36 & 27 & 9 \\
\hline Orientación al cliente & 28 & 27 & 1 \\
\hline
\end{tabular}

Fuente: elaboración propia. 
A nivel regional los resultados son más contrastantes. El Estado de México muestra los porcentajes más altos de talentos, con excepción de capacidad de aprendizaje, coraje y orientación al cliente. En sentido contrario, Hidalgo presenta los porcentajes más bajos en control del progreso, San Luis Potosí en orientación al cliente y Yucatán en capacidad de aprendizaje, decisión y discernimiento (ver tabla 3).

Tabla 3. Comparativo de talentos por estados

\begin{tabular}{|l|c|c|c|c|}
\hline Talentos & Estado de México & Hidalgo & San Luis Potosí & Yucatán \\
\hline Adaptabilidad & $90 \%$ & $50 \%$ & $71 \%$ & $80 \%$ \\
\hline Capacidad de aprendizaje & $80 \%$ & $84 \%$ & $72 \%$ & $70 \%$ \\
\hline Conciencia social & $80 \%$ & $33 \%$ & $100 \%$ & $60 \%$ \\
\hline Control del progreso & $30 \%$ & $17 \%$ & $43 \%$ & $40 \%$ \\
\hline Coraje & $70 \%$ & $33 \%$ & $71 \%$ & $80 \%$ \\
\hline Decisión & $100 \%$ & $100 \%$ & $100 \%$ & $90 \%$ \\
\hline Discernimiento & $100 \%$ & $100 \%$ & $100 \%$ & $90 \%$ \\
\hline Espíritu innovador & $60 \%$ & $33 \%$ & $57 \%$ & $40 \%$ \\
\hline Iniciativa & $80 \%$ & $67 \%$ & $57 \%$ & $70 \%$ \\
\hline Orientación al cliente & $30 \%$ & $33 \%$ & $0 \%$ & $40 \%$ \\
\hline
\end{tabular}

Fuente: elaboración propia.

\section{Discusión, conclusiones y futuras líneas de investigación}

Este artículo presentó lo resultados de un estudio descriptivo de corte cuantitativo, realizado a través de un inventario (TMA) levantado entre 33 participantes del programa Lean Startups México, todos líderes de un equipo emprendedor. Los participantes se inscribieron para ser parte del estudio; se invitó a los 60 líderes de equipos del programa y se obtuvo un total de 33 participantes que aceptaron responder el cuestionario. Estos se tomaron como muestra por conveniencia, aunque sí respondían al criterio mínimo que se calculó contaría con una muestra de 31 cuestionarios. Del total de los 33 participantes, 22 pertenecían a equipos de un grupo que fue sometido a una segunda intervención con asesoría para Construir su compañía, mientras los otros 11 solo fueron intervenidos en una primera etapa con formación 
y mentoría (al igual que los primeros 22). Como resultado se obtuvieron no solo las variaciones en los talentos de cada líder emprendedor, sino que se pudieron detectar algunas diferencias regionales en los resultados.

Los dos atributos que se encuentran mayoritariamente en ambos grupos son decisión (Grupo 1 con 95 \% versus Grupo 2 con 100 \%) y discernimiento (Grupo 1 con 95 \% versus Grupo 2 con $100 \%$ ), y pueden explicarse desde el enfoque psicológico, debido a que corresponden a la capacidad de toma de decisiones, así como a la elección entre opciones y análisis de riesgos de cada una de ellas (Martínez-Marbán, 2018); por tanto, responden al proceso continuo de toma de decisiones, asunción de riesgos (Gartner, 1988) y de éxitos y fracasos como resultado de estas (Fuentelsaz y Montero, 2015). La variación entre ambos grupos es mínima (5\%), sin embargo, es importante destacar que la menor presencia de estos dos talentos en el grupo intervenido en una segunda etapa (Grupo 1) es de llamar la atención, debido a que después de someterse al proceso, los líderes emprendedores parecen sentirse menos capaces de tomar decisiones o elegir la más indicada. Tal vez esto pueda atribuirse a que uno de los errores más comúnmente cometidos por los emprendedores al comenzar un emprendimiento por lo general es que creen saber todas las características de su producto o servicio, y parte de los aprendizajes en un proceso de implementación de metodología Lean Startup suele ser que están en un error (Blank y Dorf, 2012).

Desde otra perspectiva, de acuerdo con la postura etológica, el emprendedor es un ser que se adapta, como resultado de su aprendizaje, al desarrollarse dentro del entorno (Timbergen, 1963; Montoya, Correa y Mejía, 2009). Cobra entonces sentido que el tercer atributo predominante entre los participantes sea justamente el de adaptabilidad (Grupo 1 con 77 \% versus Grupo 2 con 73 \%), el cual, dentro de la metodología TMA, se refiere justamente a la capacidad del participante de adaptarse a las condiciones cambiantes del entorno (Martínez-Marbán, 2018). Asimismo, se podría pensar que parte de este incremento de $4 \%$ en el Grupo 1 se generó como resultado de la implementación del programa, debido a que justamente el principio de la metodología Lean Startup es entrenar al participante para navegar en ambientes inciertos (Blank y Dorf, 2012).

En lo que respecta a la capacidad de aprendizaje (Grupo 1 con 73 \% versus Grupo 2 con $82 \%$ ), se presenta una reducción de $9 \%$ en el talento, lo cual es destacable debido a que este indicador corresponde justamente a la capacidad de mostrar interés por educarse y aprender (Koellinger, 2008). Es interesante como futura línea de investigación comprender por qué este talento muestra una reducción tan amplia justamente después de haber pasado por un proceso de formación y mentoría. 
Desde la perspectiva de los indicadores más bajos, se destaca la casi nula (1 \%) variación del indicador de orientación al cliente, que se considera fundamental en la metodología Lean Startup, debido a que el centro del programa de formación y asesoría en la mentoría se enfoca en destacar la importancia de escuchar las necesidades y molestias que enfrenta el cliente con sus soluciones actuales (Blank y Dorf, 2012).

El talento que es más destacable en su variación es el de espíritu emprendedor, el cual muestra el mayor impacto después de la intervención con una reducción de $23 \%$. En este sentido, la innovación justamente se presenta como una característica fundamental del emprendedor para proponer soluciones innovadoras a las problemáticas que se enfrentan (Montoya, Correa y Mejía, 2009).

Finalmente, en el análisis de variaciones por talento hubo cambios menores a $10 \%$ en los atributos de conciencia social, iniciativa, coraje y control de progreso. Llama la atención que los que se vieron reducidos con la intervención fueron la iniciativa (4\%) y el coraje (9\%), y en sentido contrario se presentó un incremento en el control del progreso (9\%). Estos resultados contrastan con lo planteado por Blank y Dorf (2012), dado que la metodología Lean Startup a la que fueron sometidos los participantes postula que el objetivo del proceso es que el emprendedor rompa todas las suposiciones que tiene sobre las características que debería tener el producto o servicio que ofrece tras escuchar las necesidades el cliente; que tome decisiones y se adapte a ambientes inciertos y cambiantes y, donde la organización sea compleja, que presente soluciones innovadoras.

Analizando lo resultados desde una perspectiva regional, se puede identificar que los emprendedores del Estado de México presentan el mayor porcentaje en los atributos de adaptabilidad (90\%), decisión (100\%), discernimiento (100\%), espíritu emprendedor (60 \%) e iniciativa ( $80 \%)$. Asimismo, los talentos de capacidad de aprendizaje (80 \%) y conciencia social (80 \%) están en segundo sitio en comparación con los otros estados. Estos resultados pueden explicarse considerando que el mayor número de unidades económicas se encuentran ubicadas en dicha entidad (INEGI, 2020) y que por lo mismo presentan condiciones de mayor competencia y ambiente incierto que otras entidades. A esto se suma que los giros de los proyectos participantes de los emprendedores de este grupo correspondían, en su mayoría, a Edutech y sustentabilidad; ambos giros corresponden a emprendimientos que se asemejan más a los proyectos trabajados con la metodología Lean Startup en Silicon Valley (de base tecnológica y enfocado en tendencias temáticas), diseñada por Blank y Dorf (2012).

En contraste, la entidad con menores porcentajes es Hidalgo, que tiene las proporciones más bajas en los talentos de adaptabilidad (50\%), conciencia social (33\%), control del progreso (17\%), coraje (33\%) y espíritu innovador (33\%). Estos resultados se 
podrían explicar si consideramos que la entidad tiene una población menor y menos unidades económicas en comparación con otras (INEGI, 2020), lo cual reduce la competencia a la que el emprendedor se enfrenta. En el caso de los proyectos participantes en esta entidad, se registró que la mayoría correspondía a soluciones digitales para negocios, lo cual tiene sentido si se considera la vocación más industrial de la región y el requerimiento de soluciones para las empresas de la zona. Cabe señalar que el bajo porcentaje en el talento de adaptabilidad no solo puede dar respuesta a la menor competencia de la región, sino a que la entidad presenta condiciones amigables para hacer negocios, lo cual facilita con creces el proceso de emprendimiento (Banco Mundial, 2020).

En los casos particulares de San Luis Potosí y Yucatán se destaca que el primero presenta el porcentaje más bajo de orientación al cliente $(0 \%)$ y el segundo el más alto en el mismo rubro (40 \%). Ambos pueden explicarse por sus vocaciones regionales, la primera de corte industrial y la segunda de servicios, particularmente turismo (INEGI, 2020).

Una futura línea de investigación es comprender ampliamente los motivos que generaron las variaciones entre los talentos analizados, mediante un estudio de corte causal y no solo descriptivo, con la limitante de que para el presente estudio en particular se contó con una muestra por conveniencia, a la que se tuvo acceso por ser parte del programa Lean Startups México; en un análisis posterior sería necesario tener acceso a otra población.

Un estudio posterior es importante debido a que algunos de los talentos que se ven reducidos o nulamente impactados después de la intervención son justamente aquellos en los que pone énfasis la metodología Lean Startup, como es el caso del discernimiento, la decisión, la capacidad de aprendizaje, la iniciativa, el coraje, la orientación al cliente y el espíritu emprendedor. Asimismo, se abre la discusión sobre las diferencias regionales de los perfiles emprendedores, los cuales aún no se estudian ampliamente.

El presente artículo resulta una aportación relevante a la literatura, al enfocarse en el análisis del perfil de la población emprendedora mexicana, destacando diferencias regionales y ampliando la literatura existente, debido a que, como se mencionó previamente, esta se centra en países que no son de habla hispana (Luor et al., 2014). Asimismo, contrasta los resultados obtenidos en los perfiles emprendedores de los líderes de equipos intervenidos contra los aprendizajes esperados que propone Lean Startup, la metodología más utilizada para formar emprendedores en el mundo (Blank y Dorf, 2012). Dicho lo anterior, se abre la oportunidad de ampliar esta investigación en el futuro. 


\section{Referencias bibliográficas}

Banco Mundial (2020). Doing Business 2020. https://www.doingbusiness.org/content/ dam/doingBusiness/country/m/mexico/MEX.pdf

Blank, S. y Dorf B. (2012). The Startup Owner's Manual: The Step By Step, Guide for Building a Great Company. New Jersey: John Willey and Sons.

Dorf, B. (2015). Lean Startups México (archivo de audio; entrevista). México: Universidad Anáhuac/Centro Idearse.

Fuentelsaz, L. y Montero, J. (2015). «¿Qué hace que algunos emprendedores sean más innovadores?». Universia Business Review, 47: pp. 14-31.

Gartner, W. (1988). «Who is the entrepreneur? Is the wrong question». Entrepreneurship -Theory and Practice, Vol. 13 (4): 47-67.

Global Entrepreneurship Monitor, GEM (2019). GEM 2018/2019 Global Report. Recuperado en noviembre de 2019 de: https://www.gemconsortium.org/

Grupo de Desarrollo Regional del Tecnológico de Monterrey (2018). «Las megatendencias sociales actuales y su impacto en la identificación de oportunidades de negocios». FEMSA, Tecnológico de Monterrey. Recuperado en noviembre de 2018 de: https://portaldoc.itesm.mx/pls/portaldoc/docs/PORA3_1_20012012143447.pdf

Instituto Nacional de Estadística y Geografía, INEGI (2020). Censo Económico 2020. https://www.inegi.gob.mx

Iturbide, L. (2 de mayo de 2018). «La importancia del emprendimiento». El Universal. Koellinger, P. (2008). «Why are some entrepreneurs more innovative than others?». Small Business Economics, 31: 21-37.

Kurakto, D., Fisher, G. y Audretsch, D. (2020). «Unraveling the entrepreneurial mindset». Small Business Economics: 205-220. https://doi.org/10.1007/s11187020-00372-6

Lackéus, M. (2015). Education in Entrepreneurship. París: OECD.

Lean Startups México (2018). «El programa». Recuperado en noviembre de 2019 en: https://leanstartupsmexico.com.mx

Lindner, J. (2018). «Entrepreneurship Education for a Sustainable Future». Discourse \& Communication for Sustainable Education, vol. 9 (1): 115-127. https://doi.org/10. 2478/dcse-2018-0009

López, E. (5 de febrero de 2019). «Un buen emprendedor no solo se basa en tecnología». El Empresario. Recuperado en diciembre de 2019 de: http://elempresario. $\mathrm{mx} / \mathrm{emprendedores/buen-emprendedor-no-solo-se-basa-tecnologia}$

Luor, T., Lu, H., Yu, H. y Chang, K. (2014). «Trends in and contributions to entrepreneurship research: a broad review of literature from 1996 to June 2012». Scientometrics 99: 353-369. https://doi.org/10.1007/s11192-013-1203-5 
Martínez-Marbán, P. (2018). Documento oficial de Certificación de TMA 2018. México: Arancione. https://arancione.com.mx/

Montoya Marulanda, J., Correa Calle, G. y Mejía Mejía, L. (2009). «Emprendimiento: visiones desde las teorías del comportamiento humano». Revista de la Escuela de Administración de Negocios, 66: 153-168.

Murray, H. A. (1938). Explorations in personality: a clinical and experimental study of fifty men of college age. Londres: Oxford University Press.

Pickers, S. (4 de noviembre de 2015). "¿Cómo determinar el tamaño de una muestra?». Psyma. Recuperado en diciembre de 2019 de: https://www.psyma.com/ company/news/message/como-determinar-el-tamano-de-una-muestra

Rodríguez, A. (2014). «Emprendimiento, una tendencia mundial». Forbes México. https:// www.forbes.com.mx/emprendimiento-una-megatendencia-nivel-mundial/

Shaver, K. y Scott, L. (1991). «Person, Process, Choice: the Psychology of New Venture Creation». Entrepreneurship and Regional Development 27 (2): 1-47.

Solomon, G. y Fernald, L. (1993). «Innovative Approaches to Meeting Entrepreneurial Informational Needs: SBA Enters the XXI Century». Journal of Creative Behavior, vol. 27 (2): 103-111.

Schumpeter, J. (1934, 2013). Innovación y destrucción creativa. Madrid: Belloch Ediciones. Timbergen, N. (1963). «On aims and methods of ethology». Ethology, 20 (4): 410-433. Uriguen Aguirre, P., Pizarro Romero, J. y Cedeño Flores, J.E. (2018). «Metodologías de emprendimiento usadas en la universidad ecuatoriana: el caso de una institución de educación superior orense». Universidad y Sociedad, 10 (3): 309-315.

Van IJzendoorn, E., Van Weert, L., Müller, B. y Blom, A. (2010). From Talent to Performance. Utrecht: Ehrm Vision.

Vázquez-Parra, J. C. (2018). «Elementos para la valoración integral de proyectos de emprendimiento social. Una herramienta para la formación de emprendedores». Contabilidad y Negocios, 13 (26): 129-140.

Venkataraman, S. (1997). «The distinctive domain of entrepreneurship research: an editor's perspective». En J. Katz y R. Brokhaus (eds.). Advances in Entrepreneurship, Firm Emerge, and Growth. Greenwich, JAI Press, vol. 3: 119-138. 


\section{Sobre los autores}

Laura Guadalupe Iturbide Galindo es licenciada en Economía por la Universidad Anáhuac México y continuó sus estudios de maestría y doctorado en la Cornell University. Es coordinadora de la maestría en Economía y Negocios y directora del Instituto de Desarrollo Empresarial Anáhuac (IDEA) de la Universidad Anáhuac México. Tiene experiencia en análisis económico financiero en entidades públicas y privadas; ha participado en diversos consejos empresariales y asesorado organismos públicos en México. Auditora y coordinadora de proyectos con el Banco Interamericano de Desarrollo, APEC y el Pacto Mundial. Bajo su liderazgo, la Universidad Anáhuac obtuvo Mención Honorífica en el Premio Nacional del Emprendedor en 2015. Obtuvo el Premio Generación Anáhuac (2000) y el Premio Juan Huerdo, de la Asociación Mexicana de Franquicias (2019). Es coautora del libro Negocios inclusivos.

liturbid@anahuac.mx

https://orcid.org/0000-0002-6118-1779

Ricardo Rodríguez Arana Zumaya es ingeniero químico por la Universidad Nacional Autónoma de México, con maestría en Ingeniería Industrial y Ciencias de la Computación por la University of Michigan y candidato a doctor en Investigación de Operaciones en la Cornell University. Es profesor del área cuantitativa y de operaciones de la Facultad de Economía y Negocios de la Universidad Anáhuac México. Se ha desarrollado profesionalmente en el ámbito público y privado, particularmente en el área de logística, distribución y comercialización, e investigación de operaciones. Es consultor en temas económicos y de ingeniería industrial a nivel nacional e internacional. Asesor de emprendedores en temas de formulación e implementación de estrategias de negocios, modelos de factibilidad financiera y financiamiento colectivo.

ricardo.rodriguezra@anahuac.mx

https://orcid.org/0000-0002-1711-4325

Verónica Itzel López Castro es doctora en Ciencias Empresariales por la Universidad Antonio de Nebrija, con mención Cum Laude. Maestra en Economía y Negocios y licenciada en Ingeniería industrial por la Universidad Anáhuac México. Tiene amplia experiencia en el ecosistema emprendedor desde hace casi 14 años. Fue coordinadora de la Aceleradora de Empresas de la Universidad Anáhuac México Norte casi 12 años, y coordinó diversos programas de formación, mentoría, vinculación y financiamiento para emprendedores y empresarios de diversos giros. 
Ha sido panelista, ponente y moderadora en diversos eventos profesionales de emprendimiento. Actualmente es formadora, docente, investigadora y consultora en temas de emprendimiento.

itzel.lopez@anahuac.mx

https://orcid.org/0000-0002-8108-8010

Antonio Díaz Morales es doctor en Administración y Economía, así como en Mercadotecnia, por la Universidad Complutense de Madrid. Cursó el Executive MBA en la IE Business School. Ha sido profesor y directivo en diferentes instituciones educativas como EAE Business School, Nebrija Business School y la Universidad Anáhuac. Ha sido director general de empresas internacionales de diferentes mercados, como alimentos, bebidas, juguetes, entre otros. Ha publicado en diversos idiomas en sus temas de especialidad: mercadotecnia, administración y ventas. Actualmente es decano ejecutivo EIAM-PRIME en la Universidad Sergio Arboleda.

antonio.diaz@usa.edu.co

Ana Laura Aguado Pazos es licenciada en Economía por la Universidad Anáhuac México Campus Norte. Ha laborado en las áreas de mercado de capitales y análisis bursátil de Grupo Financiero Inbursa. Fue miembro de la sociedad de alumnos de la carrera de Economía y del comité del grupo de liderazgo Sinergia de la Universidad Anáhuac México. Cuenta con un artículo publicado en el periódico El Universal, titulado «El emprendedor nace o se hace» y su tesis de licenciatura versó sobre las características de los emprendedores.

ana.pazos@gmail.com 